\title{
Desenvolvimento Quantitativo e Qualitativo de Tectona grandis L.f. em Mato Grosso
}

\author{
Allan Libanio Pelissari ${ }^{1}$, Sidney Fernando Caldeira ${ }^{2}$, Ronaldo Drescher ${ }^{2}$ \\ ${ }^{1}$ Pós-graduação em Engenharia Florestal - PGEF, Universidade Federal do Paraná - UFPR, Curitiba/PR, Brasil \\ ${ }^{2}$ Faculdade de Engenharia Florestal - FENF, Universidade Federal de Mato Grosso - UFMT, Cuiabá/MT, Brasil
}

\begin{abstract}
RESUMO
O interesse no cultivo da teca para a produção de toras pode ser demonstrado pelo aumento da área plantada. Entretanto, estudos sobre o desenvolvimento da teca ainda são escassos, principalmente no Estado de Mato Grosso, em que se demonstra alta perspectiva de retorno financeiro. O objetivo deste trabalho foi avaliar o desenvolvimento quantitativo e qualitativo da teca, até dez anos de idade, em Nossa Senhora do Livramento, Mato Grosso. De 73 parcelas permanentes, foram obtidos os valores de densidade, diâmetro a $1,3 \mathrm{~m}$ do solo, alturas total e dominante, área basal, volume total, os incrementos corrente anual e médio anual, e o ajuste de modelos de regressão. Também foram avaliadas as frequências quanto a forma ou qualidade do fuste, além do estado fitossanitário. O plantio de teca apresenta desenvolvimento quantitativo de semelhante a superior aos de Ásia, África e Américas Central e do Sul, sendo que a execução dos desbastes e desramas promovem a melhoria da qualidade.
\end{abstract}

Palavras-chave: teca, reflorestamento, desbaste.

\section{Quantitative and Qualitative Development of Tectona grandis L.f. in Mato Grosso}

\begin{abstract}
The interest in the cultivation of teak for timber production can be demonstrated by the increase in planted area. However, studies on the development of teak are still scarce, especially in the state of Mato Grosso, where this cultivation presents a high prospect of financial return. In this study, we aimed to evaluate the quantitative and qualitative development of teak until ten years of age in the municipality of Nossa Senhora do Livramento, Mato Grosso state, Brazil. In an area divided in 73 permanent plots, we obtained the values of density, diameter at $1.3 \mathrm{~m}$ above the ground, total and dominant height, basal area, total volume, current annual and mean annual increments, and adjustment to regression models. We also assessed the frequency regarding the form or quality of stem and the phytosanitary status. The teak plantation studied presented quantitative development similar to or greater than those found in Asia, Africa, and South and Central Americas. The execution of thinning and pruning were the main causes of teak cultivation quality improvement.
\end{abstract}

Keywords: teak, reforestation, thinning. 


\section{INTRODUÇÃO}

A teca (Tectona grandis L.f. - Lamiaceae) é uma espécie natural da Índia, de Mianmar, da Tailândia e do Laos, e foi introduzida, há centenas de anos, na Indonésia e no Sri Lanka. Atualmente, apresenta uma distribuição relativamente ampla, sendo cultivada em diversas regiões da África e das Américas do Sul e Central, ocupando espaço de destaque no mercado entre as principais espécies produtoras de madeira tropical (Tanaka et al., 1998; Pandey \& Brown, 2000; Bermejo et al., 2004).

As florestas naturais de teca representam uma área relativamente limitada e de participação baixa na produção de madeira (Nair \& Souvannavong, 2000). Entretanto, somente a partir da proibição da exploração das florestas nativas da Índia em 1986 e do Laos e da Tailândia em 1989, os povoamentos artificiais de teca alcançaram destaque para o suprimento da demanda mundial (Pandey \& Brown, 2000), principalmente nos trópicos, em razão do maior potencial de crescimento e produtividade (Vaides et al., 2005).

O principal produto dessa espécie é a madeira utilizada em móveis de luxo e na construção naval (Figueiredo et al., 2005). A teca é considerada uma alternativa às espécies de valor econômico, como a Swietenia macrophylla King e a Torresea acreana Ducke, para o suprimento sustentável das indústrias de base florestal (Drescher, 2004). Ressalte-se, também, a possibilidade de comercialização de produtos desde os primeiros desbastes (González, 2004), o que a torna um investimento lucrativo aos seus produtores (Ângelo et al., 2009).

$\mathrm{O}$ crescimento da teca varia de acordo com as condições edáficas e climáticas locais, principalmente a precipitação, a umidade relativa e a temperatura (Sinha et al., 2011). No entanto, apresenta melhor desenvolvimento em locais com precipitação anual de $1.250 \mathrm{~mm}$ a $3.750 \mathrm{~mm}$, associada a um período de três a cinco meses de seca, e temperatura mínima de $13{ }^{\circ} \mathrm{C}$ a $17^{\circ} \mathrm{C}$ e máxima de $39^{\circ} \mathrm{C}$ a $43^{\circ} \mathrm{C}$ (Pandey \& Brown, 2000).

Essa espécie se desenvolve em uma grande variedade de solos, porém prefere os terrenos planos ou de declividade média, profundos e de boa drenagem, férteis e com $\mathrm{pH}$ ligeiramente ácido em vez de alcalino (Vásquez \& Ugalde, 1995; Centeno, 1997; Tanaka et al., 1998). É exigente em bases trocáveis, especialmente o cálcio (González, 2004), e é eficiente na utilização do fósforo (Mata, 1999); porém, é sensíveis às elevadas concentrações de alumínio trocável (Mollinedo Garcia, 2003).

As espécies florestais de crescimento rápido exigem um planejamento intensivo, por meio de intervenções silviculturais, para obter produtividade alta e madeira de qualidade (Pérez \& Kanninen, 2005). No caso da teca, a aplicação de desbastes é imprescindível para promover maior produtividade e qualidade das árvores (Garcia, 2006), pois é uma espécie exigente por luz e a competição intraespecífica, ocasionada pelo contato entre as copas, diminui a luz lateral individual (Caldeira \& Oliveira, 2008).

Normalmente, a densidade inicial dos plantios de teca é de 1.000 a 2.000 árvores.ha $^{-1}$, com o primeiro desbaste entre quatro e cinco anos de idade, e remoção de $50 \%$ dos indivíduos (Pandey \& Brown, 2000). Apesar de Garcia (2006) citar que, no Brasil, os povoamentos são implantados com 1.667 árvores. $\mathrm{ha}^{-1}$, em espaçamento de 3,0 $\mathrm{m} \times 2,0 \mathrm{~m}$ e desbastes em torno de 5, 10, 15 e 20 anos, o que proporciona entre 200 e 250 árvores.ha $^{-1}$ para o corte final, a maioria dos plantios é estabelecida no espaçamento de 3,0 $\mathrm{m} \times 3,0 \mathrm{~m}$ e, atualmente, há a tendência de $3,5 \mathrm{~m} \times 3,0 \mathrm{~m}$ e $4,0 \mathrm{~m} \times 2,5 \mathrm{~m}$, principalmente com a seleção e a clonagem de indivíduos de alto desempenho, associadas à disponibilidade de máquinas e implementos que exigem uma maior largura nas entrelinhas de plantio.

A idade de rotação dos plantios de teca em sua área de distribuição natural varia entre 50 e 90 anos (Pandey \& Brown, 2000), com produtividade de 3 a 10 $\mathrm{m}^{3} \cdot \mathrm{ha}^{-1} \cdot$ ano $^{-1}$ (Centeno, 1997), enquanto, em outras regiões, a rotação é mais curta, como na África, de 35 a 55 anos, e produtividade de 5 a $16 \mathrm{~m}^{3} \cdot \mathrm{ha}^{-1}$. ano $^{-1}$ (Dupuy et al., 1999); nas Américas do Sul e Central, com expectativa de 20 a 25 anos (Bermejo et al., 2004; González, 2010) e produtividade de 10 a 20 $\mathrm{m}^{3} \cdot$ ha $^{-1} \cdot$ ano $^{-1}$ (Centeno, 1997).

O interesse na espécie, como alternativa aos plantios florestais tradicionais, vem crescendo muito atualmente (Schuhli \& Paludzyszyn Filho, 2010), principalmente no Estado de Mato Grosso, 
em que se demonstra perspectiva alta de retorno (Shimizu et al., 2007). No entanto, apesar de diversos estudos sobre a teca no mundo, estes ainda são muito reduzidos no Brasil (Tonini et al., 2009). Com base nisso, o objetivo do presente trabalho foi avaliar o desenvolvimento quantitativo e qualitativo da teca, em plantio homogêneo, no município de Nossa Senhora do Livramento, Estado de Mato Grosso, até os dez anos de idade.

\section{MATERIAL E MÉTODOS}

O estudo foi desenvolvido em um povoamento equiâneo e homogêneo de teca, com 309 ha implantados em 1999, no espaçamento $3 \mathrm{~m} \times 3 \mathrm{~m}$, na Fazenda Campina, pertencente à empresa Teca do Brasil Ltda., localizada no município de Nossa Senhora do Livramento, Estado de Mato Grosso (Figura 1), a $73 \mathrm{~km}$ ao Sudoeste da capital Cuiabá, em uma área circunscrita à coordenada $16^{\circ} 12^{\prime} 19^{\prime \prime} \mathrm{S}$ e $56^{\circ} 23^{\prime} 00^{\prime \prime} \mathrm{W}$.

O clima da região é do tipo Aw, segundo a classificação de Köppen, com estações seca e chuvosa bem definidas (Peel et al., 2007), precipitação média de $1.300 \mathrm{~mm} . \mathrm{ano}^{-1}$, temperatura média anual de $25{ }^{\circ} \mathrm{C}$, com médias das mínimas de $20^{\circ} \mathrm{C}$ e das máximas de $32{ }^{\circ} \mathrm{C}$, e umidade relativa do ar de $70 \%$ a 75\% (Campello Júnior et al., 1991). O relevo característico é o suavemente ondulado e o solo é classificado como Planossolo Háplico Eutrófico (Embrapa, 2006), de textura francoargiloarenosa.

Para o plantio, o solo foi descompactado com subsolador, arado e nivelado. Não houve adubação de arranque, porém foram aplicados de 0,8 a 3,7 t.ha ${ }^{-1}$ de calcário magnesiano, $60 \mathrm{Kg} \cdot \mathrm{ha}^{-1}$ de fosfato e 100 a $150 \mathrm{Kg} \mathrm{ha}^{-1}$ de cloreto de potássio, aos nove anos de idade do povoamento. As desramas ocorreram a partir do segundo ano, com a retirada de galhos até um terço da altura total das árvores nessa idade, até metade no terceiro ano e até dois terços no quarto ano; a manutenção deu-se com a remoção de galhos até 7,0 $\mathrm{m}$ de altura nas idades seguintes. Os desbastes, do tipo seletivo, foram executados aos cinco e aos oitos anos, com a remoção média de $40 \%$ e 33\%, respectivamente, do número de árvores por hectare.

Para a coleta de dados, nas idades de dois a dezanos do plantio, foram alocadas 73 parcelas permanentes de $15 \mathrm{~m} \times 30 \mathrm{~m}\left(450 \mathrm{~m}^{2}\right)$, correspondendo a uma densidade inicial de 50 árvores por parcela, tendo sido mensuradas todas as árvores e obtidos os valores médios anuais de diâmetro a 1,3 m do solo (DAP) e altura total $(\mathrm{Ht})$, correspondentes às variáveis de obtenção direta no campo; também houve a determinação da altura dominante $(\mathrm{Hd})$, relacionada com a capacidade produtiva de sítios florestais e determinada pelo método de Assmann (1961); da área basal $(\mathrm{G})$, que expressa a área ocupada pelos fustes das árvores em um hectare, e do volume total $(\mathrm{Vt})$, importante para a avaliação econômica da

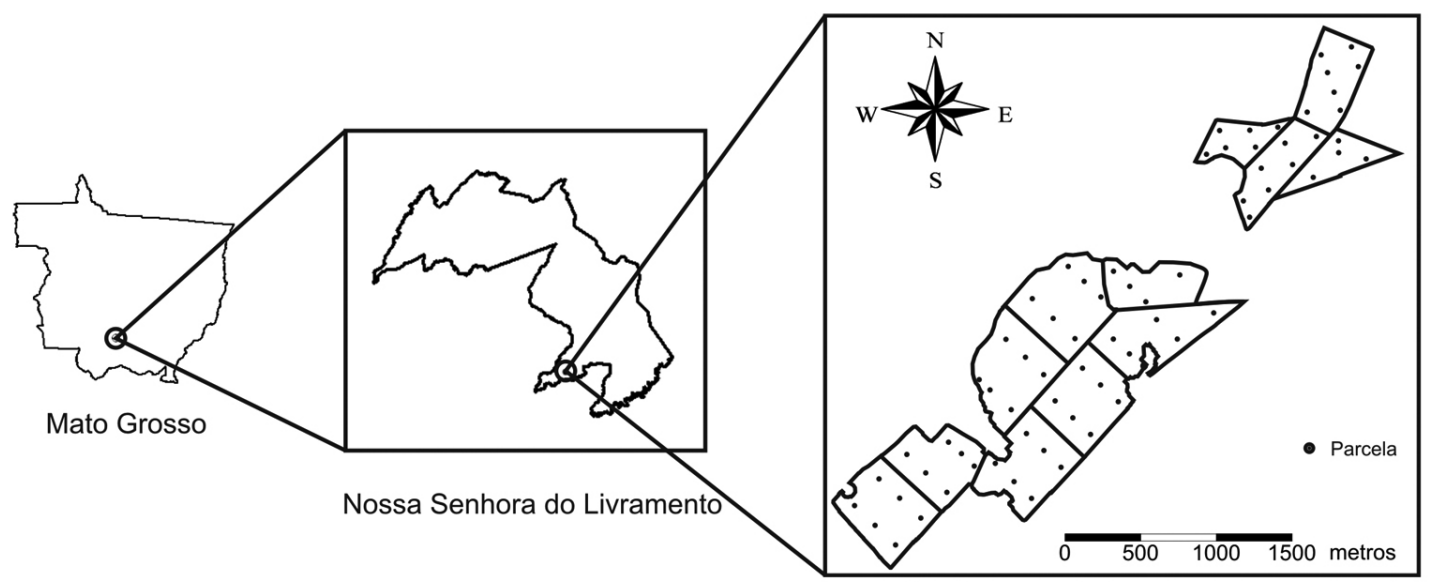

Fazenda Campina - Teca do Brasil

Figura 1. Localização do povoamento de Tectona grandis em Nossa Senhora do Livramento, Estado de Mato Grosso. Figure 1. Location of Tectona grandis stand in Nossa Senhora do Livramento, Mato Grosso State. 
produção dos povoamentos florestais, com o fator de forma artificial obtido anualmente por meio da cubagem rigorosa, com o total de 204 árvores mensuradas pelo método de Smalian. Observaramse também os incrementos corrente anual (ICA) e médio anual (IMA), calculados para as variáveis DAP, Ht, Hd, G e Vt.

Foram ajustados nove modelos de regressão (Tabela 1), disponíveis na literatura (Selle et al., 2008; Hess \& Schneider, 2010; Milani, 2010), para a estimativa das variáveis quantitativas em função da idade, com o critério de seleção em função do menor erro padrão da estimativa em porcentagem $\left(\mathrm{S}_{\mathrm{yx}} \%\right)$ e ao maior coeficiente de determinação ajustado $\left(\mathrm{R}_{\mathrm{aj} .}^{2}\right)$. Também foi analisada a significância dos coeficientes de regressão ( $\beta$ ) e a análise gráfica dos resíduos.

Para os modelos logarítmicos 1 a 7, a correção da discrepância na estimativa da variável dependente, ao se efetuar a operação inversa, foi realizada multiplicando-se a variável estimada por um fator de correção logarítmica, que corresponde a uma ferramenta estatística simples e direta para remover um viés sistemático (Sprugel, 1983). Assim, optouse pela utilização do Fator de Correção de Meyer (FCM):

\section{$F C M=e^{0,5 .(S y x)^{2}}$}

em que: $e=$ exponencial; Syx $=$ erro padrão de estimativa.

Segundo Machado et al. (2002), o erro padrão da estimativa $\left(\mathrm{S}_{\mathrm{yx}}\right)$ mede a dispersão média entre os valores observados e estimados ao longo da linha de regressão, e o coeficiente de determinação $\left(\mathrm{R}^{2}\right)$ expressa o quanto a variação total é explicada pela regressão. Quando a variável dependente sofreu alguma transformação, é necessário corrigir esses índices para possibilitar a avaliação entre as equações. No caso, foi efetuada nos modelos 1 a 7 , por meio das expressões:

$$
S y x=\sqrt{\frac{\sum_{i=1}^{n}\left(y_{i}-\hat{y}_{i}\right)^{2}}{n-p}} ; \text { e } R^{2}=1-\left(\frac{\text { SQres }}{S Q t}\right) \cdot\left(\frac{n-1}{n-p}\right)
$$

em que: yi $=$ valor observado da variável; $\hat{y} \mathrm{i}=$ valor estimado da variável; $\mathrm{n}=$ número de dados observados; $\mathrm{p}=$ número de coeficientes do modelo; SQres = soma dos quadrados dos resíduos; SQt = soma total dos quadrados.

Também foram avaliados os aspectos qualitativos do povoamento quanto à forma ou à qualidade do

Tabela 1. Modelos de regressão para o desenvolvimento de Tectona grandis em Nossa Senhora do Livramento, Estado de Mato Grosso.

Table 1. Regression models for the development of Tectona grandis in Nossa Senhora do Livramento, Mato Grosso State.

$\begin{array}{ccc}\mathbf{N}{ }^{{ }^{\circ}} & \text { Modelo } & \text { Denominação } \\ 1 & \ln (y)=\beta_{0}+\beta_{1}(1 / t) & \text { Schumacker } \\ 2 & \ln (y)=\beta_{0}+\beta_{1} \cdot \ln (t)+\beta_{2} \cdot \ln (t)^{2} & \text { Backman } \\ 3 & \ln (y)=\beta_{1} \cdot \ln (t)+\beta_{2} \cdot \ln (t)^{2} & \text { Backman modificado } \\ 4 & \ln (y)=\beta_{0}+\beta_{1} \cdot(1 / t)+\beta_{2} \cdot \ln (t) & \text { Hoerl } \\ 5 & \ln (y)=\beta_{0}+\beta_{1}(t)+\beta_{2}(t)^{2}+\beta_{3}(t)^{3} & \text { Moissev } \\ 6 & \ln (y)=\beta_{0}+\beta_{1} \cdot \ln (t)+\beta_{2}(t) & \text { Gram } \\ 7 & \ln (y)=\beta_{0}+\beta_{1}(1 / t)+\beta_{2}(1 / t)^{2}+\beta_{3}(1 / t)^{3} & \text { Spillman } \\ 8 & y=\beta_{0}\left(1-\exp \left(-\beta_{1} \cdot t\right)^{\beta 2}\right) & \text { Chapman-Richards } \\ 9 & y=\beta_{0}\left(1-\exp \left(-\beta_{1} \cdot t\right)\right) & \text { Mitscherlich }\end{array}$

$\mathrm{t}=$ idade; $\beta$ = coeficiente de regressão; $\ln =$ logaritmo neperiano; $e=$ exponencial; $\mathrm{y}=$ densidade, diâmetro a $1,3 \mathrm{~m}$ do solo, alturas total e dominante, área basal ou volume total. 
fuste, de acordo com os critérios de Jankauskis (1979), e o estado fitossanitário quanto à causa, por meio da metodologia estabelecida por Schneider et al. (1988), com os valores expressos em frequência.

\section{RESULTADOS E DISCUSSÃO}

\subsection{Desenvolvimento quantitativo}

Na Tabela 2, estão presentes os valores médios das variáveis quantitativas, entre o segundo e o décimo ano de idade do povoamento; na Figura 2, são apresentados os comportamentos estimados por meio da análise de regressão, em função da idade, e na Figura 3, os respectivos gráficos de resíduos.

$\mathrm{Na}$ primeira avaliação, aos dois anos, foi observada redução na densidade inicial de 1.111 árvores.ha ${ }^{-1}$ para 1.076 árvores.ha $^{-1}$ (Tabela 2), correspondendo a uma taxa de sobrevivência (S\%) de 96,8\% dos indivíduos, o que permaneceu aproximadamente constante até a realização do primeiro desbaste.

A taxa de sobrevivência $(\mathrm{S} \%)$ foi superior à observada por diversos autores em povoamentos

Tabela 2. Valores médios de densidade, diâmetro a 1,3 m do solo (DAP), alturas total (Ht) e dominante (Hd), área basal (G), volume total (Vt), e os seus incrementos, em função da idade, para Tectona grandis em Nossa Senhora do Livramento, Estado de Mato Grosso

Table 2. Mean values of density, diameter at $1.3 \mathrm{~m}$ above the ground (DAP), total (Ht) and dominant height (Hd), basal area $(\mathrm{G})$, total volume $(\mathrm{Vt})$, and their increments, in function of age, for Tectona grandis in Nossa Senhora do Livramento, Mato Grosso State.

\begin{tabular}{|c|c|c|c|c|c|c|}
\hline $\begin{array}{l}\text { Idade } \\
\text { (anos) }\end{array}$ & $\begin{array}{c}\text { Densidade } \\
\left(\text { árvore.ha }{ }^{-1}\right)\end{array}$ & $\begin{array}{l}\text { DAP } \\
(\mathrm{cm})\end{array}$ & $\begin{array}{l}\text { Ht } \\
(\mathrm{m})\end{array}$ & $\begin{array}{l}\text { Hd } \\
(\mathrm{m})\end{array}$ & $\begin{array}{c}\mathrm{G} \\
\left(\mathrm{m}^{2} \cdot \mathbf{h a}^{-1}\right)\end{array}$ & $\begin{array}{c}\text { Vt } \\
\left(\mathrm{m}^{3} \cdot \mathrm{ha}^{-1}\right)\end{array}$ \\
\hline 2 & 1076 & 5,21 & 4,50 & 5,43 & 2,41 & 5,92 \\
\hline 3 & 1068 & 9,25 & 8,19 & 8,79 & 7,42 & 31,64 \\
\hline 4 & 1065 & 12,02 & 10,56 & 11,03 & 12,44 & 68,12 \\
\hline 5 & 1060 & 13,76 & 12,03 & 12,16 & 16,17 & 99,03 \\
\hline 6 & 665 & 16,06 & 13,54 & 13,97 & 19,60 & 128,93 \\
\hline 7 & 662 & 17,92 & 15,46 & 16,65 & 22,80 & 167,30 \\
\hline 8 & 660 & 18,82 & 16,96 & 18,40 & 24,59 & 195,38 \\
\hline 9 & 474 & 20,69 & 17,71 & 19,01 & 26,13 & 211,37 \\
\hline 10 & 462 & 21,82 & 18,78 & 20,43 & 27,65 & 233,65 \\
\hline \multicolumn{7}{|c|}{ Incremento corrente anual } \\
\hline 2 & - & 5,21 & 4,50 & 5,43 & 2,41 & 5,92 \\
\hline 3 & - & 4,04 & 3,70 & 8,79 & 5,01 & 25,73 \\
\hline 4 & - & 2,77 & 2,36 & 11,03 & 5,02 & 36,48 \\
\hline 5 & - & 1,74 & 1,47 & 12,16 & 3,73 & 30,91 \\
\hline 6 & - & 2,30 & 1,51 & 13,97 & 3,43 & 29,90 \\
\hline 7 & - & 1,86 & 1,92 & 16,65 & 3,20 & 38,37 \\
\hline 8 & - & 0,91 & 1,51 & 18,40 & 1,79 & 28,09 \\
\hline 9 & - & 1,86 & 0,75 & 19,01 & 1,54 & 15,99 \\
\hline 10 & - & 1,13 & 1,06 & 20,43 & 1,51 & 22,28 \\
\hline \multicolumn{7}{|c|}{ Incremento médio anual } \\
\hline 2 & - & 2,61 & 2,25 & 2,71 & 1,21 & 2,96 \\
\hline 3 & - & 3,08 & 2,73 & 2,93 & 2,47 & 10,55 \\
\hline 4 & - & 3,00 & 2,64 & 2,76 & 3,11 & 17,03 \\
\hline 5 & - & 2,75 & 2,41 & 2,43 & 3,23 & 19,81 \\
\hline 6 & - & 2,68 & 2,26 & 2,33 & 3,27 & 21,49 \\
\hline 7 & - & 2,56 & 2,21 & 2,38 & 3,26 & 23,90 \\
\hline 8 & - & 2,35 & 2,12 & 2,30 & 3,07 & 24,42 \\
\hline 9 & - & 2,30 & 1,97 & 2,11 & 2,90 & 23,49 \\
\hline 10 & - & 2,18 & 1,88 & 2,04 & 2,76 & 23,37 \\
\hline
\end{tabular}


(A)

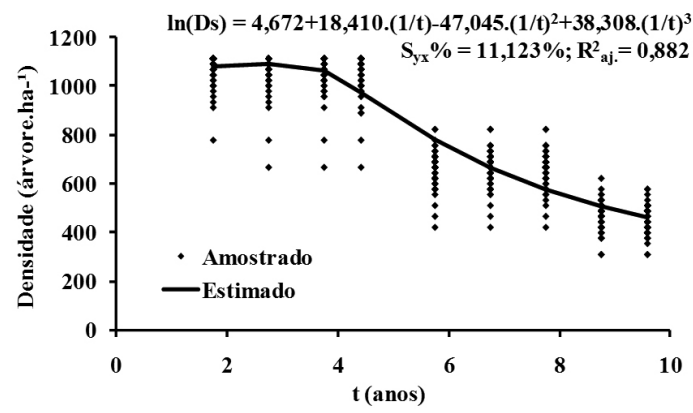

(C)

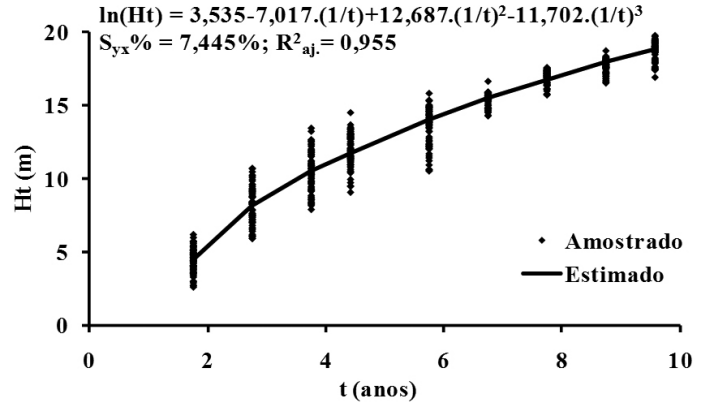

(E)

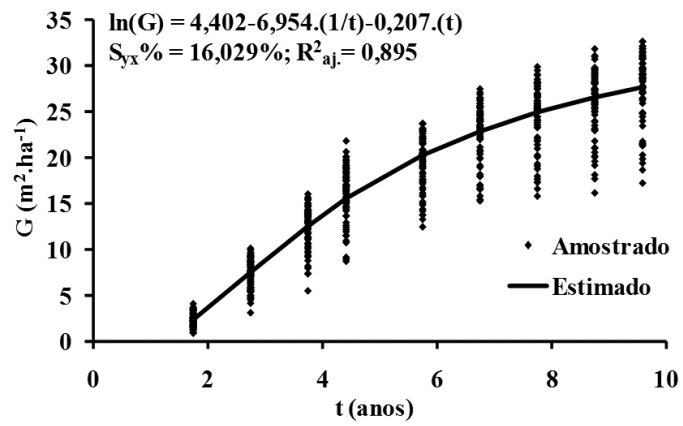

(B)

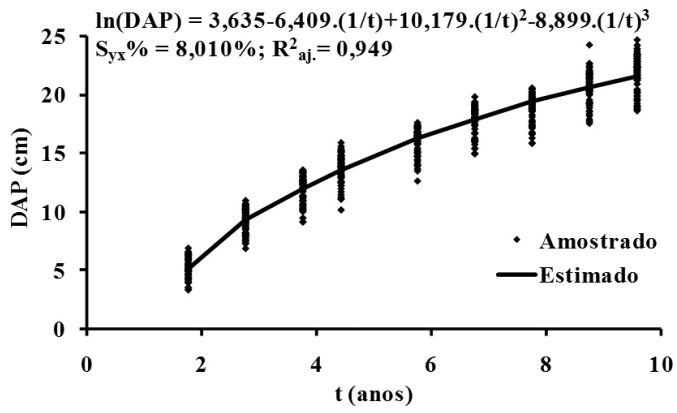

(D)

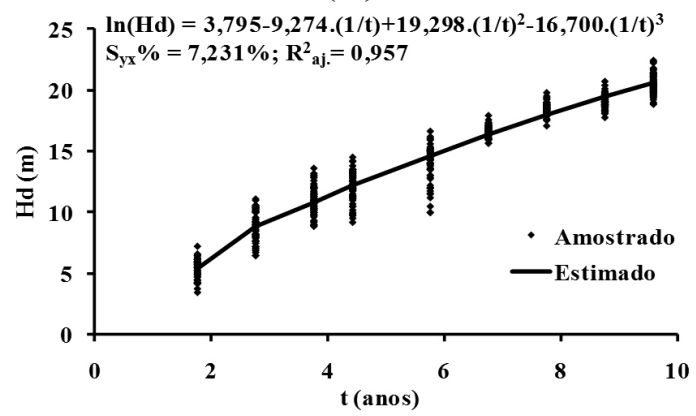

(F)

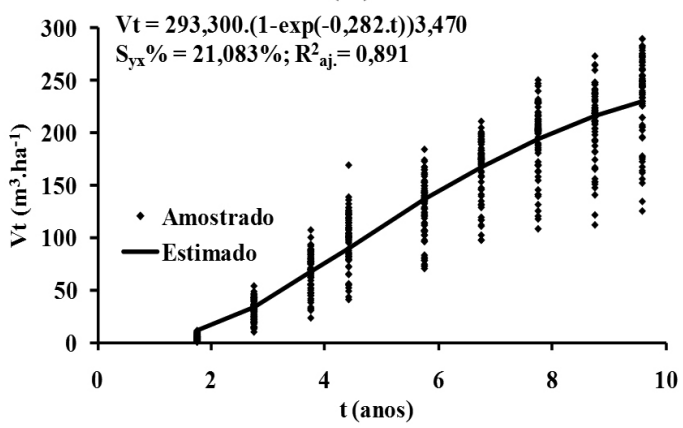

Figura 2. Comportamento estimado de densidade (A), diâmetro a $1,3 \mathrm{~m}$ do solo (B), altura total (C), altura dominante (D), área basal (E) e volume total (F), em função da idade, para Tectona grandis, em Nossa Senhora do Livramento, Estado de Mato Grosso.

Figure 2. Behavior estimated of the density (A), diameter at $1.3 \mathrm{~m}$ above the ground (B), total height (C), dominant height (D), basal area (E) and total volume (F), in function of age, for Tectona grandis in Nossa Senhora do Livramento, Mato Grosso State.

de teca, como: Catie (1986) em Honduras, com S\% de 77\%; Macedo et al. (2005) no Noroeste do Estado de Minas Gerais, com S\% igual a 70,4\%; Ribeiro et al. (2006) em Minas Gerais, em diferentes níveis de adubação e com S\% entre 63,8\% e 90,0\%, e Flores et al. (2009) no México, com S\% de 86,32\%.

De acordo com Macedo et al. (2005), a porcentagem de sobrevivência das espécies florestais pós-plantio no campo fornece informações sobre o potencial de adaptação dessas espécies em um determinado local. Assim, a taxa de sobrevivência observada demonstra uma adequada adaptabilidade da teca às condições edafoclimáticas da região de Nossa Senhora do Livramento-MT.

O modelo de Spillman, por meio da equação ln $($ Densidade $)=4,672+18,410 .(1 / t)-47,045 \cdot(1 / t)^{2}+$ 38,308. $(1 / t)^{3}$, forneceu o melhor ajuste da densidade em função da idade (Figura 2A), com erro-padrão de estimativa $\left(\mathrm{S}_{\mathrm{yx}} \%\right)$ de $11,123 \%$ e coeficiente de determinação ajustado $\left(\mathrm{R}^{2}{ }_{\text {aj. }}\right)$ igual a 0,882 , além de 
(A)

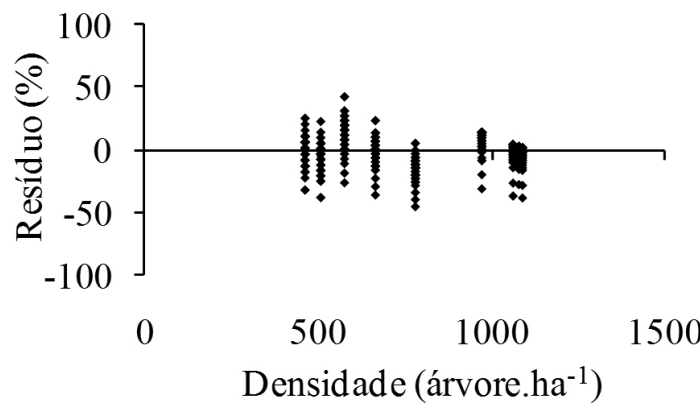

(C)

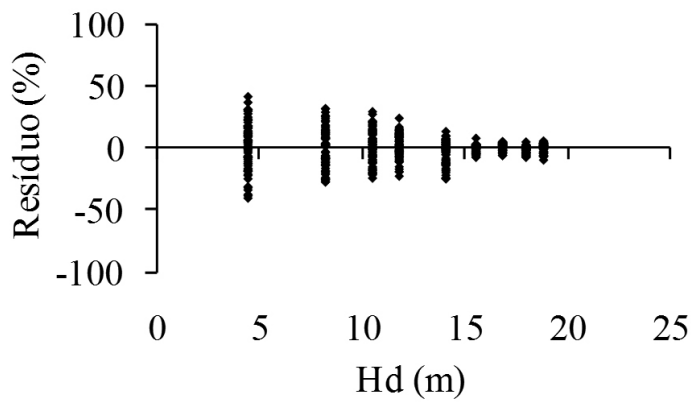

(E)

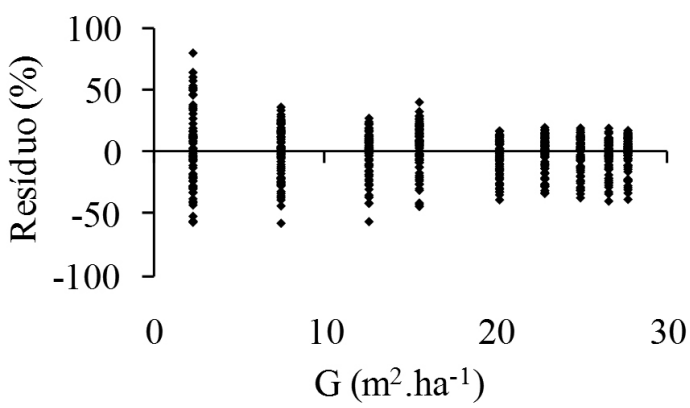

(B)

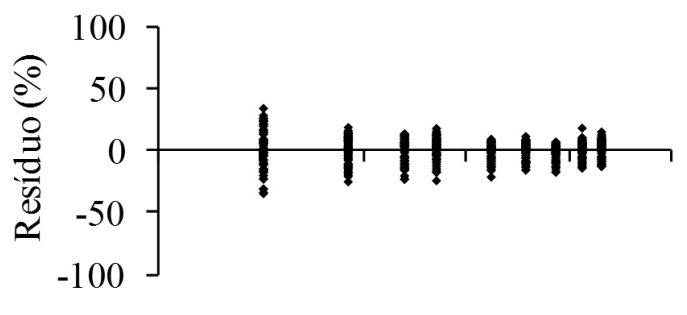

(D)

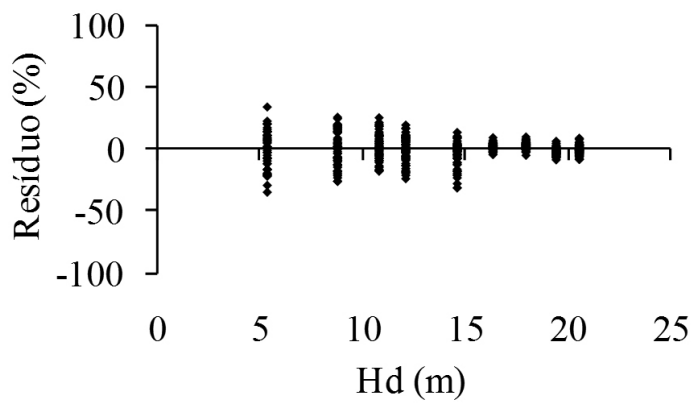

(F)

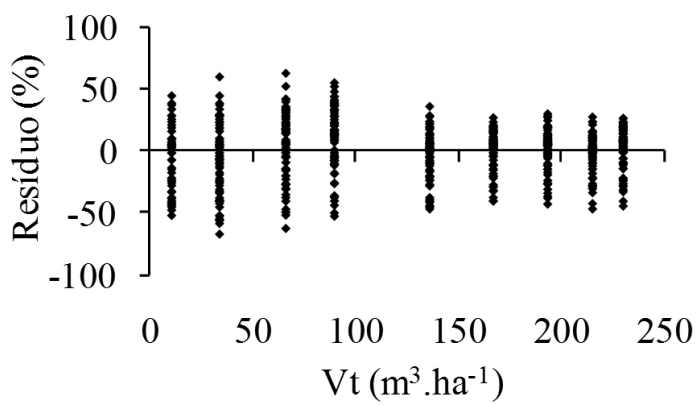

Figura 3. Distribuição dos resíduos para a estimativa de densidade (A), diâmetro a 1,3 m do solo (B), altura total (C), altura dominante (D), área basal (E) e volume total (F), em função da idade, para Tectona grandis, em Nossa Senhora do Livramento, Estado de Mato Grosso.

Figure 3. Distribution of residuals from estimates of the density (A), diameter at $1.3 \mathrm{~m}$ above the ground (B), total height (C), dominant height (D), basal area (E) and total volume (F), in function of age, for Tectona grandis in Nossa Senhora do Livramento, Mato Grosso State.

coeficientes de regressão significativos e dispersão residual adequada (Figura 3A).

Em geral, os valores do DAP (Tabela 2) foram superiores aos observados na Ásia, em povoamentos de teca na Indonésia (Bailey \& Harjanto, 2005) e na Índia (Shukla, 2009); na África, em Gana (Nunifu \& Murchison, 1999) e Tanzânia (Bekker et al., 2004); na América Central, no Panamá (Montero, 1995; Mollinedo et al., 2005), e no Brasil, em Mato Grosso (Oliveira, 2003; Garcia, 2006) e Roraima
(Tonini et al., 2009). Tais valores também foram semelhantes a diversos plantios da Costa Rica (Vallejos Barra, 1996; Kanninen et al., 2004).

Foi observado um acentuado decréscimo no incremento corrente anual em DAP até os cinco anos, seguido por uma elevação aos seis anos (Tabela 2), após a aplicação do desbaste. Comportamento semelhante foi observado do sexto ao oitavo ano de idade, com elevação desse valor, aos nove anos, após o segundo desbaste. Garcia (2006) destaca 
que a variável DAP responde proporcionalmente às intensidades do desbaste aplicado em povoamentos de teca, estabelecendo-se a seguinte relação: quanto maior a sua intensidade, maior o ganho em DAP.

$\mathrm{O}$ incremento médio anual em DAP apresentou, inicialmente, um acréscimo de 2,61 para 3,08 $\mathrm{cm}$ ano $^{-1}$ aos três anos (Tabela 2), seguido por uma constante redução; note-se que tal incremento não se mostrou influenciado pelos desbastes. Em geral, forneceu valores superiores aos observados nos plantios de teca em Roraima (Tonini et al., 2009) e semelhantes aos melhores sítios da Guatemala (Vaides et al., 2005; Castellanos, 2006) e aos de média qualidade do México (González, 2010).

A equação $\ln (D A P)=3,635$ - 6,409. $(1 / t)+10,179$. $(1 / t)^{2}$ - 8,899. $(1 / t)^{3}$, ajustada pelo modelo de Spillman, descreveu com precisão o progressivo aumento do DAP em função da idade (Figura 2B), fornecendo um $\mathrm{S}_{\mathrm{yx}} \%$ de $8,010 \%, \mathrm{R}_{\text {aj. }}^{2}$ igual a 0,949 , coeficientes de regressão significativos e ausência de tendenciosidade pela análise gráfica dos resíduos (Figura 3B).

A altura total (Ht), indicada na Tabela 2, apresentou valores superiores aos observados em Gana (Nunifu \& Murchison, 1999); no Brasil, em Minas Gerais (Macedo et al., 2005) e Roraima (Tonini et al., 2009), e na Índia (Shukla, 2009). Foram semelhantes aos sítios de média a alta qualidade da Costa do Marfim (Dupuy et al., 1999); Tanzânia (Bekker et al., 2004); Costa Rica (Bermejo et al., 2004); em Mato Grosso, Brasil (Drescher, 2004), e Panamá (Mollinedo et al., 2005).

O primeiro desbaste, inicialmente, influenciou pouco o incremento corrente em altura total (Tabela 2), assim como observado por Garcia (2006), em povoamentos de teca em Sinop-MT, e por Tonini et al. (2009), em Roraima. Entretanto, aos seis anos, após a segunda intervenção, não houve resposta ao crescimento em altura, somente dois anos após os desbastes. Segundo Andrade et al. (2007), os incrementos iniciais podem ser lentos após os desbastes e a retomada do crescimento ocorre assim que as árvores finalizam a adaptação do sistema radicular e da arquitetura das copas à nova configuração espacial. Além disso, González (2004) afirma que a teca responde aos desbastes favorecendo mais o incremento em diâmetro do que em altura.
$\mathrm{O}$ incremento médio anual em altura total apresentou uma elevação de 2,25 para 2,73 m.ano ${ }^{-1}$ aos três anos, seguido por uma constante redução (Tabela 2). A aplicação do primeiro desbaste forneceu um incremento médio anual de $1,51 \mathrm{~m} \cdot$ ano $^{-1}$ aos cinco anos, semelhante ao observado por Kanninen et al. (2004), ao desbastarem $40 \%$ da densidade em um povoamento de teca na Costa Rica. Em geral, os valores foram superiores aos observados nos melhores sítios da Guatemala (Castellanos, 2006) e semelhantes aos de média qualidade da Costa Rica (Vaides et al., 2005) e do México (González, 2010).

$\mathrm{O}$ modelo de Spillman forneceu o melhor ajuste a partir da equação $\ln (H t)=3,535-7,017$. $(1 / t)+12,687 .(1 / t)^{2}-11,702 .(1 / t)^{3}$ e descreveu o crescimento contínuo da altura total em função da idade (Figura 2C), com $\mathrm{S}_{\mathrm{yx}} \%$ igual a $7,445 \%, \mathrm{R}_{\text {aj. }}^{2}$ de 0,955 , coeficientes de regressão significativos e ausência de tendenciosidade no gráfico residual (Figura 3C).

Os valores das alturas dominantes (Hd), indicados na Tabela 2, foram superiores aos obtidos em plantios de teca no Panamá (Mollinedo et al., 2005) e no México (González, 2010), e semelhantes aos observados em sítios de média a alta qualidade da Costa do Marfim (Dupuy et al., 1999), de Gana (Nunifu \& Murchison, 1999), da Costa Rica (Bermejo et al., 2004), da Guatemala (Vaides et al., 2005) e de Mato Grosso, Brasil (Drescher, 2004; Novaes, 2009).

$\mathrm{O}$ incremento corrente anual em altura dominante apresentou forte redução até os cinco anos, com elevação após a execução do primeiro desbaste (Tabela 2). Entretanto, houve redução após o segundo desbaste, com acréscimo somente dois anos depois. Tal fato pode estar relacionado com o caráter seletivo do desbaste, no qual possivelmente foram retirados indivíduos de maior porte, porém com danos fitossanitários ou formas de fuste de baixa qualidade.

$\mathrm{O}$ incremento médio anual em altura dominante apresentou baixa oscilação dos valores ao longo do tempo (Tabela 2), o que demonstra que a altura dominante é pouco influenciada por tratamentos silviculturais e pela competição (Tonini et al., 2002). Os valores foram superiores aos observados por 
Vásquez \& Ugalde (1995) nos melhores sítios de Guanacaste, Costa Rica.

A trajetória crescente da altura dominante, em função da idade (Figura 2D), foi mais bem estimada pelo modelo de Spillman, com a equação $\ln (H d)=$ 3,795 - 9,274. $(1 / t)+19,298 .(1 / t)^{2}-16,700 .(1 / t)^{3}$, e apresentou um $\mathrm{S}_{\mathrm{yx}} \%$ igual a $7,231 \%, \mathrm{R}^{2}$ aj. de 0,957 , coeficientes de regressão significativos e ausência de tendenciosidade pela análise dos resíduos (Figura 3D).

Os valores da área basal $(\mathrm{G})$, apresentados na Tabela 2, foram semelhantes aos observados por Drescher (2004) em plantios de teca com até dez anos de idade nos sítios de média qualidade de Santo Antônio de Leverger e Brasnorte, no Estado de Mato Grosso. Esse fato corrobora com Pérez \& Kanninen (2005), que afirmam que, em geral, a teca apresenta um crescimento superior nas Américas Central e do Sul, em relação a outras regiões tropicais.

A redução do incremento corrente anual em área basal aos cinco anos (Tabela 2) indica os efeitos da competição no povoamento, visto que a área basal, em povoamentos de teca, é fortemente afetada pela competição intraespecífica (Hernández et al., 1993). Note-se que essa competição pode proporcionar a redução da capacidade fotossintética, causada pela limitação da disponibilidade de luz, água e nutrientes (Schneider \& Finger, 1994).

$\mathrm{O}$ incremento médio anual em área basal apresentou, inicialmente, um aumento com a idade e, posteriormente, um declínio (Tabela 2). Os seus valores foram superiores aos observados nos melhores sítios do Panamá (Mollinedo et al., 2005), da Guatemala (Vaides et al., 2005; Castellanos, 2006) e do México (González, 2010).

Após o primeiro desbaste, aos seis e sete anos, os incrementos corrente anual e médio anual, em área basal, apresentaram valores semelhantes (Tabela 2), indicando o momento da área basal ótima do povoamento, em que é obtido o maior incremento periódico em volume em um determinado período de crescimento (Assmann, 1961).

O modelo de Hoerl, por meio da equação ln $(G)=4,402-6,954 .(1 / t)-0,207 .(t)$, proporcionou uma boa precisão, com $\mathrm{S}_{\mathrm{yx}} \%$ de $16,029 \%, \mathrm{R}_{\text {aj. }}^{2}$ de 0,895, coeficientes de regressão significativos e ausência de tendências nos resíduos (Figura 3E). O ajuste demonstrou que a área basal apresentou um crescimento inicial mais acentuado, seguido por uma tendência de redução (Figura 2E).

O volume total (Vt), apresentado na Tabela 2, foi superior aos valores médios observados em povoamentos de teca com diferentes idades em Gana (Nunifu \& Murchison, 1999); Malásia (Noor, 2003); Panamá (Montero, 1995; Griess \& Knoke, 2011), e Brasil, nos Estados do Acre (Figueiredo et al., 2005), de Minas Gerais (Macedo et al., 2005) e de Roraima (Tonini et al., 2009). Foram superiores aos melhores sítios da Costa do Marfim (Dupuy et al., 1999) e da Costa Rica (Bermejo et al., 2004), e semelhantes aos sítios de média qualidade de Santo Antônio de Leverger e Brasnorte, Estado de Mato Grosso (Drescher, 2004).

$\mathrm{O}$ incremento corrente anual em volume total aos seis e nove anos apresentou redução após a execução dos desbastes e, somente dois anos após a intervenção, houve ganho (Tabela 2). Glufke et al. (1997) informaram que essa variável está em função da qualidade do sítio florestal e que o efeito do desbaste é concentrar o incremento em um número menor de árvores e não aumentá-lo. Em um primeiro momento, os desbastes reduzem a produção em volume do povoamento, mas esta redução é compensada pelo valor econômico agregado com o aumento da dimensão das árvores remanescentes (Schneider \& Finger, 1994).

$\mathrm{O}$ incremento médio em volume total mostrou, inicialmente, um comportamento crescente e com tendência de estabilização ao final do período de estudo (Tabela 2). Segundo Vaides et al. (2005), o incremento médio é útil para interpretar o crescimento da teca. Em geral, os valores foram superiores aos dos sítios de alta qualidade de Gana (Nunifu \& Murchison, 1999), Costa Rica (Vásquez \& Ugalde, 1995; Bermejo et al., 2004), Panamá (Mollinedo et al., 2005), Guatemala (Castellanos, 2006) e México (González, 2010).

A estimativa do volume total, em função da idade, foi mais bem delineada pelo modelo de Chapman-Richards, com a equação $\ln (V i)=$ $293,300$. (1-exp $(-0,282 . t))^{3,470}, \mathrm{~S}_{\mathrm{yx}} \%$ igual a $21,083 \%$, $\mathrm{R}^{2}$ aj. de 0,891 , coeficientes de regressão significativos e ausência de tendenciosidade no gráfico de resíduos (Figura 3F). O ajuste demonstrou um crescimento 
inicial lento até aproximadamente três anos, seguido por um progressivo aumento e encerrando com uma leve tendência de decréscimo da curva (Figura 2F).

\subsection{Desenvolvimento qualitativo}

$\mathrm{Na}$ Figura 4, estão representadas as frequências médias das árvores de teca quanto aos parâmetros qualitativos de forma ou qualidade do fuste, e do estado fitossanitário quanto à causa, ambos em função da idade.

Aproximadamente $90 \%$ dos indivíduos aos dois anos de idade apresentaram forma de fuste de classe FF1 (Figura 4A), correspondendo a árvores com fuste reto e sem galhos laterais. No entanto, esse percentual foi reduzido para $53 \%$ aos três anos, com $42 \%$ dos indivíduos na classe FF2, de fuste reto e galhos laterais.

A presença de galhos laterais nessa fase de crescimento pode estar relacionada com a ação dos ventos, associada a indivíduos com alta relação altura/ DAP, ou seja, árvores de maior porte, porém com baixo ganho em diâmetro, sendo mais suscetíveis a inclinarem ao serem submetidas a fortes ventos e sofrerem rachaduras no fuste. Observa-se, também, que, ao serem expostas à insolação direta da luz na casca, estimula-se a formação de gemas laterais e, posteriormente, de galhos nos locais fendidos. É comum observar árvores inclinadas a partir dos dois anos de idade e esse quadro se intensificando aos três anos. Vásquez \& Ugalde (1995) ressaltam que a teca é muito afetada por ventos fortes, enquanto Lamprecht (1990) destaca que é comum o surgimento de ramos adventícios e que isso justifica a aplicação da desrama sistemática em plantios de teca.

Aos quatro anos, houve aumento na frequência de indivíduos na classe FF3, com alguma tortuosidade e sem galhos laterais. Nessa idade, foi constatado melhora na qualidade dos fustes, com 64,3\% na classe FF1, tendência que se repetiu aos cinco anos, com 76,4\% dos indivíduos na classe FF1. Já aos seis anos, houve redução significativa da frequência da classe FF3, fato relacionado com a aplicação do desbaste baixo e seletivo, cuja eficiência na melhora da qualidade do povoamento foi observada pelo restante do período avaliado.

As alterações na forma do fuste ao longo do tempo observadas no presente trabalho são destacadas por Friedl (1989), o qual afirma que a mudança da forma das árvores em povoamentos florestais jovens é rápida, enquanto que, nas idades avançadas, as alterações são lentas e tendem a se estabilizar; tal fato está relacionado com as diferentes classes de copas e as posições sociológicas dentro de um mesmo povoamento. Assim, a competição por luz exerce grande influência quanto à tortuosidade, pois as árvores tendem a procurá-la quando são submetidas à concorrência. Dessa forma, é comum a presença de fustes tortuosos, em mais de um plano, e voltados na direção em que as copas estão expostas à luminosidade (Mattos, 2002).
(A)

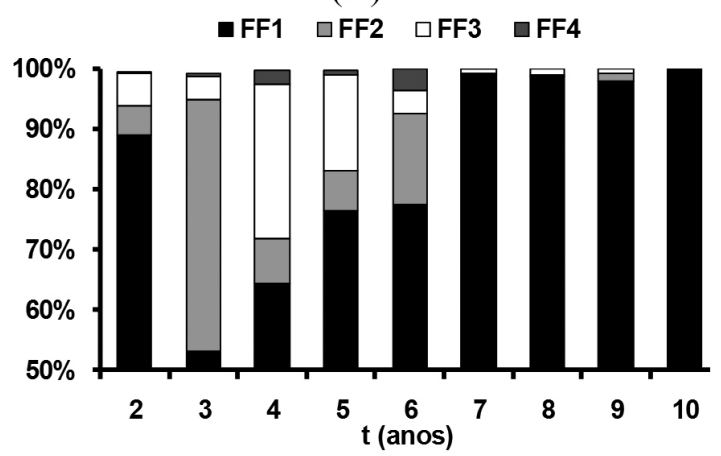

(B)

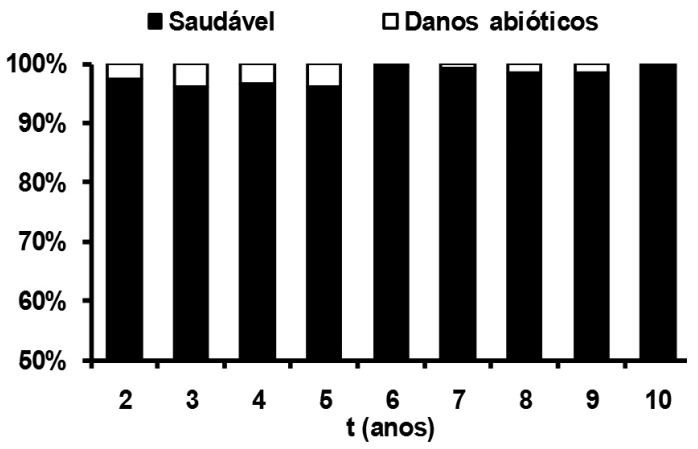

Figura 4. Variação da forma e qualidade do fuste (A) e do estado fitossanitário (B), em função da idade, para Tectona grandis, em Nossa Senhora do Livramento, Estado de Mato Grosso.

Figure 4. Variation of the shape and quality of the stem (A) and phytosanitary state (B), in function of age, for Tectona grandis in Nossa Senhora do Livramento, Mato Grosso State. 
Quanto ao estado fitossanitário (Figura 4B), mais de 95\% dos indivíduos mostraram-se saudáveis ao longo do período de estudo. Entretanto, até $4 \%$ apresentaram danos abióticos entre os dois e cinco anos de idade. Em sua maioria, os danos foram caracterizados como rachaduras no fuste, os quais foram suprimidos a partir da retirada desses indivíduos por meio dos desbastes.

\section{CONCLUSÕES}

O plantio de teca no município de Nossa Senhora do Livramento, Estado de Mato Grosso, apresenta desenvolvimento quantitativo de semelhante a superior aos plantios da Ásia, da África e das Américas Central e do Sul, sendo que a execução dos desbastes e as desramas promovem a melhoria da qualidade do povoamento florestal.

\section{STATUS DA SUBMISSÃO}

Recebido: $17 / 01 / 2013$

Aceito: 04/08/2013

Publicado: 30/09/2013

\section{AUTOR(ES) PARA CORRESPONDÊNCIA}

\section{Allan Libanio Pelissari}

Programa de Pós-graduação em Engenharia Florestal, Faculdade de Engenharia Florestal, Universidade Federal do Paraná - UFPR, Av. Prefeito Lothário Meissner, 900, Jardim Botânico - Campus III, CEP 80210-170, Curitiba, PR, Brasil

e-mail: allanpelissari@gmail.com

\section{Sidney Fernando Caldeira}

Faculdade de Engenharia Florestal, Universidade Federal de Mato Grosso - UFMT, Av. Fernando Corrêa da Costa, 2367, Boa Esperança, CEP 78125-070, Cuiabá, MT, Brasil e-mail: sidneycal@gmail.com

\section{REFERENNCIAS}

Andrade CM, Finger CAG, Thomas C, Schneider PR. Variação do incremento anual ao longo do fuste de Pinus taeda L. em diferentes idades e densidades populacionais. Ciência Florestal 2007; 17(3): 239-246.
Ângelo H, Silva VSM, Souza ÁN, Gatto AC. Aspectos financeiros da produção de teca no Estado de Mato Grosso. Floresta 2009; 39(1): 23-32.

Assmann E. The principles of forest yield study. New York: Pergamon Press; 1961. 506 p. PMid:13684851.

Bailey JD, Harjanto NA. Teak (Tectona grandis L.) tree growth, stem quality and health in coppiced plantations in Java, Indonesia. New Forests 2005; 30: 55-65. http:// dx.doi.org/10.1007/s11056-004-1116-5

Bekker C, Rance W, Monteuuis O. Teak in Tanzania: II. The Kilombero Valley Teak Company. Bois et Forêts des Tropiques 2004; 279(1): 11-21.

Bermejo I, Cañellas I, San Miguel A. Growth and yield models for teak plantations in Costa Rica. Forest Ecology and Management 2004; 189: 97-110. http://dx.doi. org/10.1016/j.foreco.2003.07.031

Caldeira SF, Oliveira DLC. Desbaste seletivo em povoamentos de Tectona grandis com diferentes idades. Acta Amazonica 2008; 38(2): 223-228. http://dx.doi. org/10.1590/S0044-59672008000200005

Campello Júnior JH, Priante Filho $\mathrm{N}$, Caseiro FT. Caracterização macroclimática de Cuiabá. In: Anais do III Encontro Nacional de Estudos sobre o Meio Ambiente; 1991; Londrina. Londrina: UEL/NEMA; 1991. p. 542 552.

Castellanos AFR. Efecto del establecimiento de plantaciones forestales de Teca (Tectona grandis L.f.) en áreas de potrero sobre las características del suelo en Petén, Guatemala [dissertação]. Turrialba: Centro Agronómico Tropical de Investigación y Enseñanza; 2006.

Centro Agronómico Tropical de Investigación y Enseñanza - Catie. Silvicultura de espécie promisorias para producción de leña en América Central: resultados de cinco años de investigación. Turrialba: CATIE; 1986. $228 \mathrm{p}$.

Centeno JC. El manejo de las plantaciones de teca. Actualidad Forestal Tropical 1997; 5(2): 10-12.

Drescher R. Crescimento e produção de Tectona grandis Linn. F., em povoamentos jovens de duas regióes do Estado de Mato Grosso [tese]. Santa Maria: Universidade Federal de Santa Maria; 2004

Dupuy B, Maitre H, Kanga AN. Table de production du teck (Tectona grandis): L'exemple de la Côte d'Ivoire. Bois et Forêts des Tropiques 1999; 261(3): 5-16.

Embrapa. Centro Nacional de Pesquisa de Solos. Sistema Brasileiro de Classificação de Solos. 2. ed. Rio de Janeiro: Embrapa; 2006. 306 p.

Figueiredo EO, Oliveira AD, Scolforo JRS. Análise econômica de povoamentos não desbastados de Tectona grandis L.f., na microrregião do baixo Rio Acre. Cerne 2005; 11(4): 342-353. 
Flores HJM, Ávalos VMC, Sánchez JJG, Che MB. Evaluación de una plantación de tres especies tropicales de rápido crecimiento en Nuevo Urecho, Michoacán. Revista Ciencia Forestal en México 2009; 34: 61-87.

Friedl RA. Dinâmica da forma dos fustes em povoamentos plantados de Araucária angustifólia (Bert) O. Ktze [dissertação]. Curitiba: Universidade Federal do Paraná; 1989.

Garcia ML. Intensidade de desbaste em um povoamento de Tectona grandis L.f., no município de Sinop - MT [dissertação]. Cuiabá: Universidade Federal de Mato Grosso; 2006.

Glufke C, Finger CAG, Schneider PR. Crescimento de Pinus elliottii Engelm sob diferentes intensidades de desbaste. Ciência Florestal 1997; 7(1): 11-25.

González SAS. Relación del suelo con el crecimiento inicial y contenido foliar de teca (Tectona grandis), $y$ adaptación de leguminosas para control de arvenses bajo un sistema fertirriego en Campeche, México [dissertação]. Turrialba: Centro Agronómico Tropical de Investigación y Enseñanza; 2010.

González WF. Manual para productores de teca (Tectona grandis L. f) en Costa Rica. Costa Rica: Heredia; 2004. $121 \mathrm{p}$.

Griess VC, Knoke T. Can native tree species plantations in Panama compete with Teak plantations? An economic estimation. New Forests 2011; 41: 13-39. http://dx.doi. org/10.1007/s11056-010-9207-y

Hernández R, Torres A, Márquez $\mathrm{O}$, Franco W. Contenido foliar de nutrimentos y crecimiento en plantaciones de teca en Ticoporo, Ven. Turrialba 1993; 43(1): 11-15.

Hess AF, Schneider PR. Crescimento em volume de Araucaria angustifolia (Bertol.) Kuntze em três locais do Rio Grande do Sul, Brasil. Ciência Florestal 2010; 20(1): 107-122.

Jankauskis J. Recuperação de florestas tropicais mecanicamente exploradas. Belém: SUDAM; 1979. 58 p.

Kanninen M, Pérez D, Montero M, Víquez E. Intensity and timing of the first thinning of Tectona grandis plantations in Costa Rica: results of a thinning trial. Forest Ecology and Management 2004; 203: 89-99. http://dx.doi.org/10.1016/j.foreco.2004.07.054

Lamprecht H. Silvicultura nos trópicos: ecossistemas florestais e respectivas espécies arbóreas. Rossdorf: TZVerl.-Ges.; 1990. 343 p.

Macedo RLG, Gomes JE, Venturin N, Salgado BG. Desenvolvimento inicial de Tectona grandis L.f. (teca) em diferentes espaçamentos no município de Paracatu, MG. Cerne 2005; 11(1): 61-69.

Machado SA, Conceição MB, Figueiredo DJ. Modelagem do volume individual para diferentes idades e regimes de desbaste em plantações de Pinus oocarpa. Ciências Exatas e Naturais 2002; 4(2): 185-196.

Mata MM. Factores de sitio que influyen en el crecimiento de Tectona grandis L. f. y Bombacopsis quinata (Jacq.) Dugand, en Costa Rica [dissertação]. Valdivia: Universidad Austral de Chile; 1999.

Mattos RB. Características qualitativas e possibilidade de ganho de fuste em espécies euxilóforas nativas da região central do Rio Grande do Sul [dissertação]. Santa Maria: Universidade Federal de Santa Maria; 2002.

Milani EJ. Crescimento de Podocarpus lambertii Klotzsch ex Endl. em duas regiões fitogeográficas no Estado do Rio Grande do Sul [tese]. Santa Maria: Universidade Federal de Santa Maria; 2010.

Mollinedo Garcia MS. Relación suelo-planta, factores de sitio y respuesta a la fertilización, en plantaciones jóvenes de teca (Tectona grandis L. f.), en la zona Oeste, Cuenca del canal de Panamá [dissertação]. Turrialba: Centro Agronómico Tropical de Investigación; 2003.

Mollinedo M, Ugalde L, Alvarado A, Verjans JM, Rudy LC. Relación suelo-árbol y factores de sitio, en plantaciones jóvenes de teca (Tectona grandis), en la Zona Oeste de la Cuenca del Canal de Panamá. Agronomía Costarricense 2005; 29(1):67-75.

Montero M. Dinámica de crecimiento de teca (Tectona grandis) bajo fertilización en El Limón de Chupampa, Herrera, Panamá. In: Anais do I Seminario Técnico sobre Fertilización Forestal; 1995; Santiago. Veraguas: CATIE/ INRENARE; 1995. p. 17-29.

Nair CTS, Souvannavong O. Emerging research issues in the management of teak. Unasylva 2000; 51:45-54.

Noor HM. Growth of teak (Tectona grandis) on lateritic soil at Mata Ayer Forest Reserve, Perlis. Journal of Tropical Forest Science 2003; 15(1):190-198.

Novaes DM. Modelagem de crescimento e produção de povoamentos de Tectona grandis L.f. a partir de análise de tronco [dissertação]. Viçosa: Universidade Federal de Viçosa; 2009.

Nunifu TK, Murchison HG. Provisional yield models of Teak (Tectona grandis Linn F.) plantations in northern Ghana. Forest Ecology and Management 1999; 120: 171178. http://dx.doi.org/10.1016/S0378-1127(98)00529-5

Oliveira JRV. Sistema para cálculo de balanço nutricional e recomendação de calagem e adubação de povoamentos de teca - Nutriteca [dissertação]. Viçosa: Universidade Federal de Viçosa; 2003.

Pandey D, Brown C. Teak: a global overview. Unasylva 2000; 51:3-13.

Peel MC, Finlayson BL, McMahon TA. Updated world map of the Köppen-Geiger climate classification. Hydrology Earth System Sciences 2007; 11: 1633-1644. http://dx.doi.org/10.5194/hess-11-1633-2007 
Pérez D, Kanninen M. Stand growth scenarios for Tectona grandis plantations in Costa Rica. Forest Ecology and Management 2005; 210: 25-441. http:// dx.doi.org/10.1016/j.foreco.2005.02.037

Ribeiro FA, Macedo RLG, Venturim N, Morais VM, Gomes JE, Yoshitami M Jr. Feitos da adubação de plantio sobre o estabelecimento de mudas de Tectona grandis L.f. (teca). Revista Científica Eletrônica de Engenharia Florestal 2006; 4(7): 1-13.

Schneider PR, Brena DA, Finger CAG. Manual para a coleta de informações dendrométricas. Santa Maria: UFSM/CEPEF/FATEC; 1988. 28 p.

Schneider PR, Finger CAG. Determinação de regimes de desbaste para povoamentos de Pinus elliottii E. do Planalto Ocidental no Estado de Santa Catarina. Ciência Florestal 1994; 4(1): 43-59.

Schuhli GS, Paludzyszyn Filho E. O cenário da silvicultura de teca e perspectivas para o melhoramento genético. Pesquisa Florestal Brasileira 2010; 30: 217-230. http://dx.doi.org/10.4336/2010.pfb.30.63.217

Selle GL, Pauleski DT, Braz EM. Como classificar sítios florestais através da altura dominante do povoamento. Colombo: Embrapa Florestas; 2008. 46 p.

Shimizu JY, Klein H, Oliveira JRV. Diagnóstico das plantações florestais em Mato Grosso. Cuiabá: Central de Texto; $2007.63 \mathrm{p}$.

Shukla PK. Nutrient dynamics of Teak plantations and their impact on soil productivity - A case study from India. In: Proceedings of the XIII World Forestry Congress; 2009; Buenos Aires. Buenos Aires; 2009. 11 p.
Sinha SK, Deepak MS, Rao RV, Borgaonkar HP. Dendroclimatic analysis of teak (Tectona grandis L. f.) annual rings from two locations of peninsular India. Current Science 2011; 100(1):84-88.

Sprugel DG. Correcting for bias in log-transformed allometric equations. Ecology 1983; 64(1):209-210. http://dx.doi.org/10.2307/1937343

Tanaka N, Hamazaki T, Vacharangkura T. Distribution, Growth and site requirements of teak. Japan Agricultural Research Quarterly 1998; 32:65-77.

Tonini H, Costa MCGC, Schwengber LAM. Crescimento da teca (Tectona grandis) em reflorestamento na Amazônia Setentrional. Pesquisa Florestal Brasileira 2009; 59:05-14.

Tonini H, Finger CAG, Schneider PR, Spathelf P. Comparação gráfica entre curvas de índice de sítio para Pinus elliottii e Pinus taeda desenvolvidas no Sul do Brasil. Ciência Florestal 2002; 12(1):143-152.

Vaides E, Ugalde L, Galloway G. Crecimiento y productividad de teca en plantaciones forestales jóvenes en Guatemala. Recursos Naturales y Ambiente 2005; 46:137-145.

Vallejos Barra OS. Productividad y relaciones del índice de sitio con variables fisiográficas, edafoclimáticas y foliares para Tectona grandis L.F., Bombacopsis quinatum (Jacq.) Dugand y Gmelina arborea Roxb. en Costa Rica [dissertação]. Turrialba: Centro Agronómico Tropical de Investigación; 1996.

Vásquez WC, Ugalde LAA. Rendimiento y calidad de sitio para Gmelina arborea, Tectona grandis, Bombacopsis quinatum y Pinus caribaea en Guanacaste, Costa Rica. Turrialba: CATIE; 1995.40 p. 